\title{
CSF chitinase proteins in amyotrophic lateral sclerosis
}

Alexander G Thompson ${ }^{1+}$

Elizabeth Gray ${ }^{1+}$

Alexander Bampton ${ }^{1}$

Dominika Raciborska ${ }^{2}$

Kevin Talbot ${ }^{1}$

Martin R Turner ${ }^{1}$

${ }^{1}$ Nuffield Department of Clinical Neurosciences, University of Oxford, Oxford, UK

${ }^{2}$ Barts and the London School of Medicine, London, UK

${ }^{+}$These authors contributed equally

Correspondence: Professor Martin Turner

Clinical Neurosciences

West Wing Level 6

John Radcliffe Hospital

Oxford OX3 9DU

Tel: +44 (0)1865 223380

martin.turner@ndcn.ox.ac.uk

\section{Keywords}

Amyotrophic lateral sclerosis; neuroinflammation; microglia; biomarker

Title 49 characters

\section{Word Count}

Main (excluding abstract, figure legends, tables and table legends): 3431

Abstract 250; Introduction 551; Discussion 751; Main 3431; Figures 4; Colour figures 4;

Supplementary Figures 2; Tables 4

\section{References 24}




\section{Licence statement}

I, the Submitting Author has the right to grant and does grant on behalf of all authors of the Work (as defined in the below author licence), an exclusive licence and/or a non-exclusive licence for contributions from authors who are: i) UK Crown employees; ii) where BMJ has agreed a CC-BY licence shall apply, and/or iii) in accordance with the terms applicable for US Federal Government officers or employees acting as part of their official duties; on a worldwide, perpetual, irrevocable, royalty-free basis to BMJ Publishing Group Ltd (“BMJ”) its licensees and where the relevant Journal is co-owned by BMJ to the co-owners of the Journal, to publish the Work in Journal of Neurology, Neurosurgery, and Psychiatry and any other BMJ products and to exploit all rights, as set out in our licence.

The Submitting Author accepts and understands that any supply made under these terms is made by BMJ to the Submitting Author unless you are acting as an employee on behalf of your employer or a postgraduate student of an affiliated institution which is paying any applicable article publishing charge (“APC”) for Open Access articles. Where the Submitting Author wishes to make the Work available on an Open Access basis (and intends to pay the relevant APC), the terms of reuse of such Open Access shall be governed by a Creative Commons licence - details of these licences and which Creative Commons licence will apply to this Work are set out in our licence referred to above. 


\section{Objective}

To evaluate the classifier performance, clinical and biochemical correlations of CSF levels of the chitinase proteins Chitotriosidase-1 (CHIT1), Chitinase-3-like protein 1 (CHI3L1) and Chitinase-3-like protein 2 in amyotrophic lateral sclerosis (ALS).

\section{Methods}

CSF levels of CHIT1, CHI3L1, CHI3L2, phosphorylated neurofilament heavy chain (pNFH) and C-reactive protein (CRP) were measured by ELISA in a longitudinal cohort of patients with ALS ( $n=82)$, primary lateral sclerosis (PLS, $n=10)$, ALS-mimic conditions $(n=12)$, healthy controls $(\mathrm{n}=25)$ and asymptomatic carriers of ALS-causing genetic mutations (AGC; $n=5)$.

\section{Results}

CSF CHIT1, CHI3L1 and CHI3L2 were elevated in ALS patients compared with healthy controls $(p<0.001)$ and ALS-mimics (CHIT1 $p<0.001$; CHI3L1 $p=0.017$; CHI3L2 $p<0.001$ ). CHIT1 and CHI3L2 were elevated in ALS compared with PLS (CHIT1 $p=0.021$; CHI3L1 $p=0.417$; CHI3L2 $p<0.001$ ). Chitinase levels were similar in AGCs and healthy controls.

Chitinase proteins distinguished ALS from healthy controls (AUC: CHIT1 0.92; CHI3L1 0.80; CHI3L2 0.90), mimics (AUC: CHIT1 0.84; CHI3L1 0.73; CHI3L2 0.88) and, to a lesser extent, PLS (AUC: CHIT 0.73; CHI3L1 0.51; CHI3L2 0.82) but did not outperform pNFH.

CHIT1 and CHI3L2 correlated with disease progression rate (Pearson's $r=0.49, p<0.001$; $r=0.42, p<0.001$ respectively). CHI3L1 correlated with degree of cognitive dysfunction ( $r=-$ $0.25, p=0.038$ ). All chitinases correlated with pNFH. CHIT1 levels were associated with survival in multivariate models. Chitinase levels were longitudinally stable.

\section{Conclusions}

CSF chitinase proteins may have limited value as independent diagnostic and stratification biomarkers in ALS, but offer a window into non-autonomous mechanisms of motor neuronal loss in ALS, specifically in assessing response to therapies targeting neuroinflammatory pathways. 


\section{INTRODUCTION}

Amyotrophic lateral sclerosis (ALS), the most common form of motor neuron disease (MND), is a fatal neurodegenerative disease resulting in progressive weakness and death due to loss of neurons of the motor cortex, brainstem and spinal cord ${ }^{1}$. Despite two licensed disease-modifying treatments, most patients survive less than 3 years from symptom onset ${ }^{2}$, but there is significant clinical and prognostic heterogeneity. Characteristic proteinaceous inclusions of the DNA and RNA binding protein TDP-43 are found in more than $95 \%$ of cases post mortem ${ }^{3}$. A significant proportion of ALS cases are associated with frontotemporal cognitive and behavioural dysfunction. Although most cases occur sporadically, up to $10 \%$ are associated with a family history of ALS or frontotemporal dementia (FTD) ${ }^{4}$. The most common genetic cause of both ALS and FTD is a hexanucleotide repeat expansion (HRE) in an intronic region of the C9orf72 gene ${ }^{6}$.

In common with other neurodegenerative diseases, glial pathology is observed in ALS ${ }^{7}$. Although the role of microglia and astrocytes in ALS is unresolved, whether causing, exacerbating or mitigating neurodegeneration, interest in modifying glial mechanisms as a therapeutic approach has led to clinical trials of drugs aiming to suppress microglial activity. Biomarkers quantifying the glial response in patients are therefore a priority to assess target engagement in therapeutic trials. Cerebrospinal fluid (CSF) levels of chitinase proteins, in particular chitotriosidase 1 (CHIT1), chitinase 3-like protein 1 (CHI3L1 or YKL-40) and chitinase 3-like protein 2 (CHI3L2 or YKL-39), may be markers of acivity of microglial (and possibly other glia, such as astrocytes) in $\mathrm{ALS}^{8-10}$. Both agnostic and target-driven studies of CSF have highlighted elevations of these three proteins in ALS as well as other neurodegenerative diseases including Alzheimer disease and frontotemporal dementia ${ }^{11} 12$.

This study sought to compare levels of CSF chitinase proteins in patients with ALS, those with diseases that clinically mimic ALS, patients with the upper motor neuron-only slowlyprogressive variant primary lateral sclerosis (PLS), healthy control subjects and asymptomatic carriers of the C9orf72 hexanucleotide repeat expansion. Diagnostic performance of chitinase proteins was examined in relation to CSF levels of one of the leading ALS diagnostic biomarker candidates, namely phosphorylated neurofilament heavy chain (pNFH). Chitinase proteins are also compared with another ALS biomarker candidate, CSF C-reactive (CRP). This marker has been previously shown to be elevated in CSF from ALS patients and, though not validated as a measure of intrathecal inflammation, likely reflects a combination of serum CRP level and blood-CSF barrier dysfunction ${ }^{13} 14$.

\section{METHODS}

\section{Participants and sampling}

Participants were recruited from a tertiary ALS referral clinic at the John Radcliffe Hospital, Oxford, UK. Patients were diagnosed with ALS and other motor disorders by two experienced neurologists (KT, MRT). Consecutively consenting ALS and PLS patients were recruited as well as patients with non-ALS causes of motor weakness in whom the diagnosis of ALS was considered a plausible differential diagnosis (designated ALS mimics). Healthy control subjects were spouses and friends of clinic attendees. Known C9orf72 HRE carriers (asymptomatic gene carriers, AGC) had chosen to have presymptomatic genetic testing prior to and entirely independently of this study through a Clinical Geneticist-led pathway. 
CSF samples were obtained by lumbar puncture directly into polypropylene collection tubes. Venous blood was obtained using the BD Vacutainer ${ }^{\circledR}$ Safety-Lok ${ }^{\mathrm{TM}}$ blood collection set into $5.0 \mathrm{~mL}$ serum separator tubes. Biofluid samples were centrifuged at 3000rpm for 10 minutes at $4^{\circ} \mathrm{C}$ within one hour of sampling and stored at $-80^{\circ} \mathrm{C}$ until measurement. Samples were obtained at the first visit and then at intervals of 3-6 months. ALS and PLS patients were followed until death.

Clinical parameters were obtained contemporaneously on the day of biofluid sampling. Symptom onset was defined as first weakness or relevant neurological symptom reported by patients. Disability was assessed using the revised ALS functional rating scale (ALSFRS-R) at each visit ${ }^{15}$, and the Edinburgh Cognitive and Behavioural ALS Screen (ECAS) at first visit $^{16}$. Disability progression rate (PR) was calculated by dividing the gradient of descent of the ALSFRS-R from symptom onset to sampling. A clinical upper motor neuron burden score was calculated by the presence of hyperreflexia in each of 15 reflexes obtained at the first visit ${ }^{17}$.

Ethical approval for the study was obtained from NRES Central Committee South Central Berkshire (14/SC/0083 and 10/H0505/71). All participants provided written consent (or gave permission for a carer to sign on their behalf).

\section{Biochemical assays}

All ELISAs and activity assays were performed in duplicate. Plates were read using a FLUOstar Omega plate reader (BMG LABTECH, UK). Standard curves were fitted with 4parameter logistic regression using MARS data analysis software (BMG LABTECH, UK). CSF samples were thawed on ice prior to measurement. Measurements of CSF and serum protein concentrations and activity were performed using commercially-available assays according to the manufactures instructions (CircuLex ${ }^{\mathrm{TM}}$ human Chitotriosidase ELISA, CircuLex $^{\mathrm{TM}}$ human YKL-39 ELISA, CycLex ${ }^{\circledR}$ Chitotriosidase Fluorimetric Assay Kit, MBL, UK; Human Chitinase 3-like 1 Quantikine ELISA kit, R\&D systems, UK; Abcam ${ }^{\circledR}$ CRP human ELISA kit, Abcam ${ }^{\circledR}$, UK; pNf-H ELISA, Euroimmun AG, Germany).

Samples were diluted where necessary to achieve a concentration within the linear range of standard curve measurements. Median intra- and interassay coefficients of variation (CV) were below 20\% and for all assays (CHIT1 intra-assay median 2.54\% [IQR 1.38-5.52\%], interassay 7.20\% [3.54-12.86\%], LLOQ 70.95pg/mL; CHI3L1 1.86\% [0.90-3.61\%], 2.80\% [2.70-5.90\%], LLOQ 100.02pg/mL; CHI3L2 2.61\% [127-5.20], 5.07\% [4.05-5.10\%], LLOQ 38.34pg/mL; CRP 4.67\% [2.12-8.00\%], 17.22\% [11.81-22.64], LLOQ 75.81pg/mL; pNFH 2.54\% [1.38-5.52\%], 7.20\% [3.54-12.86\%] LLOQ 0.32ng/mL; CHIT1 activity 2.38\% [1.51$5.61 \%], 13.90 \%)$.

\section{Statistical analysis}

All hypothesis tests were two-sided, using $p<0.05$ to denote statistical significance. Analysis of demographics was performed using a Chi ${ }^{2}$ test with post hoc Fisher exact test for categorical variables and Kruskall-Wallis $H$ test with Dunn's post hoc for continuous variables. Group comparisons of level were performed using pairwise Mann-Whitney $U$ tests with multiple comparison correction using the Benjamini-Hochberg step-up procedure. Additional ANCOVA of log-transformed analyte concentration was performed to correct for age differences between groups. Classifier performance was examined using receiver operator characteristic (ROC) analysis. Comparison between ROC curves was performed using the DeLong method, with paired analysis to account for the use of multiple markers in 
the same samples. Optimal combinations were established by the highest ROC of summed log abundances of all combinations of chitinase proteins.

Univariate and multivariate correlation analysis was performed using simple and multiple linear regression and associations between predictor and outcome variables were tested using a Student's $t$-test. Chitinase protein concentrations were log transformed prior to inclusion in linear regression models. Survival analysis was performed using a Log-rank test for individual variables and Cox proportional hazards modelling incorporating clinical variables that have been previously associated with survival in ALS and for which sufficient information was available ${ }^{18}$. Longitudinal analysis was performed using a random intercept, random slope linear mixed effects model, with individual participants specified as random effects. Statistical analysis was performed in R.

\section{RESULTS}

\section{Demographic data}

Demographic data is given in Table 1. Subject age differed by group ( $p=0.011)$. Post hoc testing demonstrated significant differences between AGCs and other groups (ALS $p=0.013$, PLS $p=0.010$, mimics $p=0.047$, healthy controls $p=0.043$ ). There were significantly more male subjects in the ALS and mimic groups compared with other groups on post hoc testing (ALS vs PLS $p=0.037$, healthy controls $p=0.037$, AGCs $p=0.037$; mimic vs PLS $p=0.037$, healthy controls $p=0.037$, AGCs $p=0.037$ ).

\section{Cross-sectional chitinase protein levels}

Cross-sectional chitinase protein levels are given in Table 2. CSF levels of CHIT1, CHI3L1 and CHI3L2 were elevated in the first-visit CSF samples of ALS patients compared to healthy controls (FDR-adjusted $p<0.001$ for all chitinase proteins), mimics (CHIT1 $p<0.001$, CHI3L1 $p=0.017$, CHI3L $2 p<0.001$ ) and AGCs (CHIT1 $p=0.003$, CHI3L1 $p<0.001$, CHI3L2 $p=0.002)$. Levels of CHIT1 and CHI3L2 were elevated in ALS compared to PLS, though levels of CHI3L1 were similar in both ALS and PLS (CHIT1 $p=0.021$, CHI3L1 $p=0.417$, CHI3L2 $p<0.001$; Figure 1A-C).

Levels of all three chitinase proteins were similar between healthy control subjects and AGCs (CHIT1 $p=0.844$, CHI3L1 $p=0.657$, CHI3L2 $p=0.578$ ). Using ANCOVA to control for the differing age of participants across groups yielded similar results, with the exception of CHI3L1 levels which were higher in ALS patients than PLS patients and higher in healthy controls compared with AGCs (ALS vs PLS $p=0.027$, healthy control vs AGC $p=0.027$;

\section{Supplementary table 1).}

\section{Classifier performance}

Chitinase protein diagnostic performance is outlined in Figure 1D-F. All chitinase proteins performed well in distinguishing ALS from healthy controls and mimics. Individual chitinase proteins did not outperform pNFH alone, nor did the optimal combination of chitinase proteins and pNFH (ALS vs healthy controls: CHIT1 AUC 0.92 [95\% CI 0.86-0.98], CHI3L1 0.80 [0.70-0.91], CHI3L2 0.90 [0.84-0.96], pNFH 0.97 [0.94-1.00], CHIT1 plus pNFH 0.97 [0.94-1.00], difference between AUC of $\mathrm{pNFH}$ and $\mathrm{pNFH}$ plus CHIT1 $p=0.841$; mimic CHIT1 0.84 [0.72-0.95], CHI3L1 0.73 [0.58-0.88], CHI3L2 0.88 [0.81-0.95], pNFH 0.91 
[0.83-0.99], CHI3L2 plus pNFH 0.94 [0.88-1.00], difference between AUC of pNFH and pNFH plus CHI3L2 $p=0.608)$.

Performance distinguishing ALS from PLS was worse, particularly using CHI3L1 (CHIT1 0.74 [0.55-0.93], CHI3L1 0.58 [0.36-0.79], CHI3L2 0.78 [0.65-0.91], pNFH 0.88 [0.780.97], CHI3L2 plus pNFH 0.88 [0.78-0.98], difference between AUC of pNFH and pNFH plus CHI3L2 $p=0.973)$.

\section{Correlation with clinical parameters}

\section{Disease progression rate}

In first visit samples from patients with ALS and PLS, linear regression models of $\log _{10} \mathrm{PR}$ were constructed. Higher levels of CHIT1 and CHI3L2 were modestly correlated with higher PR in both simple linear regression and multiple linear regression models controlling for age of onset, site of onset and gender (CHIT1 simple regression Pearson's $r=0.49, p<0.001$, multiple regression $p=0.049$; CHI3L2 $r=0.42, p<0.001$, multiple regression $p=0.048$; Figure 2A and 2C. Multiple regression models are detailed in Table 3). CHI3L1 levels did not correlate with the rate of disease progression in simple or multiple regression models (simple regression $r=0.13, p=0.241$; multiple regression $p=0.176$; Figure $2 \mathbf{B}$ and Table 3). Levels of pNFH were more closely correlated with $\log _{10} \mathrm{PR}$ (simple regression $r=0.69, p<0.001$; multupled regression $p<0.001$; Table 3, Figure 2D).

\section{Cognitive function}

Regression analysis was also performed on a subgroup of patients $(n=39)$ with ALS and PLS who had undergone testing using the ECAS. Higher levels of CHI3L1 were found to be weakly associated with a greater degree of cognitive dysfunction as measured by the ALSspecific ECAS score in both simple linear regression and multiple linear regression models controlling for the effect of disease duration and the degree of disability as measured by the ALSFRS-R (Pearson's $r=-0.25, p=0.038$; multiple regression $p=0.044$; Figure 2D). CHIT1, CHI3L2 and pNFH levels were not associated with ALS-specific ECAS score in simple or multiple regression models (CHIT1 $r=-0.02, p=0.910$, multiple regression $p=0.811$; CHI3L2 $r=-0.02, p=0.906$, multiple regression $p=0.967$; pNFH $r=-0.07, p=0.706$, multiple regression $p=0.98$; Figure $2 \mathrm{E}, \mathbf{G}, \mathbf{H})$.

\section{Upper motor neuron signs}

Similar regression analysis was performed to correlate chitinase levels with the burden of UMN signs. Higher CHI3L1 was weakly associated with higher UMN score in multiple linear regression models controlling for the degree of disability as measured by the ALSFRS$\mathrm{R}$ and the disease duration, though not in simple linear regression (simple regression Pearson's $r=0.21, p=0.064$; multiple regression $p=0.048$, Figure $2 \mathbf{H}$ ); there was no association between CHIT1, CHI3L2 or pNFH and UMN score in either simple or multiple regression models (CHIT1 $r=0.11, p=0.284$, multiple regression $p=0.284$; CHI3L2 $r=-0.08$, $p=0.459$, multiple regression $p=0.442$; pNFH $r=0.07, p=0.540$, multiple regression $p=0.138$; Figure 2I, K, L).

\section{Biochemical parameters}




\section{Chitotriosidase activity}

In order to test whether the CHIT1 detected in CSF is active, CSF chitotriosidase activity was measured with a fluorimetric activity assay. Chitotriosidase activity was highly correlated with CHIT1 levels (data from all visits; Pearson's $r=0.93, p<0.001$; Supplementary Figure 1A), suggesting that elevated levels of CHIT1 do not constitute inactive enzyme.

\section{Chitinase proteins}

Levels of the three chitinase proteins were moderately correlated (data from all visits; CHIT1-CHI3L1 Pearson's $r=0.49, p<0.001$; CHIT1-CHI3L2 Pearson's $r=0.49, p<0.001$; CHI3L1-CHI3L2 Pearson's $r=0.47, p<0.001$; Supplementary Figure 1B).

\section{pNFH}

Levels of the chitinase proteins were moderately correlated with the axonal degeneration marker pNFH, with CHIT1 most closely correlated, followed by CHI3L2 and CHI3L1 (CHIT1 Pearson's $r=0.70, p<0.001$; CHI3L1 $r=0.58, p<0.001$; CHI3L2 $r=0.61, p<0.001$; Supplementary Figure 1C-E).

\section{CRP}

A weak correlation between CSF levels of the acute phase protein CRP and only CHI3L1 was observed (data for all visits; CHIT1 Pearson's $r=-0.03, p=0.647$; CHI3L1 $r=0.24$, $p<0.001$; CHI3L2 $r=0.05, p=0.523$; Supplementary Figure 1F-H).

\section{Longitudinal analysis}

Longitudinal CSF chitinase protein levels in ALS and PLS patients were fitted to a random intercept random slope linear mixed effects model, beginning at the baseline visit. The number of samples included in the longitudinal analysis is summarised in Table 1. Subjects were stratified into high- or low-PR categories dependent upon whether they were above or below (or equal to) the median PR at the baseline visit. There was no significant rise in the levels of any of the chitinase proteins over time whether stratified by PR or not (Figure 3).

\section{Survival analysis}

In univariate analysis, no significant association of any of the chitinase proteins and survival of ALS patients was observed (CHIT1 $p=0.075$; CHI3L1 $p=0.922$; CHI3L2 $p=0.239$; survival curves are illustrated in Supplementary Figure 2). A Cox proportional hazards model was created, incorporating several factors known to be associated with shortened survival in ALS (age of symptom onset, site of onset and gender) with chitinase proteins. Several additional factors, known to be associated with survival including respiratory function and C9orf72 status were not included since the data was not available. This model identified a significant association of elevated CHIT1 (as well as older age of onset) with increased hazard of death (CHIT1 HR=11.684, 95\% CI 2.620-52.101, $p=0.001$ ). On inclusion of pNFH in the model, there remained significant associations of CHIT1 and age with survival (CHIT1 HR=6.831, 95\% CI 1.135-105.765, $p=0.021$; Table 4). Cox proportional hazards modelling using the same clinical parameters with the chitinase proteins and pNFH examined individually 
indicated significant associations of CHIT1 (HR 4.844, $p=0.004$ ) and pNFH (HR 1.386, $p=0.004$; Supplementary Table 2).

\section{Serum chitinase proteins}

Serum chitinase protein levels were measured in a cross-sectional subset of 20 ALS patients and 20 healthy control subjects. A weak correlation between CHIT1 levels in CSF and serum was observed, though there was no correlation between CSF and serum levels of CHI3L1 or CHI3L2 (CHIT1 Pearson's $r=0.41, p=0.012$; CHI3L1 $r=0.09, p=0.593$; CHI3L2 $r=0.00$, $p=0.982$; Figure 4A-C). No significant difference between serum levels of ALS patients and healthy controls was observed for any of the three chitinase proteins (Figure 4D-F).

\section{DISCUSSION}

This study compared CSF glial markers from a cohort of patients with ALS, disease mimics and relevant control groups, including longitudinal data and in relation to clinical parameters of ALS and other CSF markers.

CHIT1 and CHI3L1 in ALS: CHIT1 and CHI3L1 were elevated in patients with ALS compared with both healthy controls and asymptomatic carriers of genetic mutations predisposing to ALS. CHIT1 and CHI3L2 showed the best performance in distinguishing ALS from disease mimics and PLS. In common with previous studies, however, they did not improve upon the performance of neurofilament proteins. The size of the disease mimic and PLS groups and differences in the sampling latency with respect to PLS limits extrapolation of this finding to the clinical diagnostic setting. Truly accurate ascertainment of diagnostic accuracy would require prospective recruitment of a large number of subjects in whom the diagnosis is not established at the time of sampling. Although a 2016 study using dried blood spot CHIT1 activity, however, did demonstrate differences between healthy controls and correlation with progression rate, the poor correlation between CSF and serum levels of the chitinase proteins agrees with recent analysis suggesting that measurement of serum chitinase proteins is unlikely to be useful as an ALS biomarker ${ }^{9} 11$.

Lower levels (and activity) of CHIT1 has been shown in subjects with a common duplication in the CHIT1 gene, found in around a third of Europeans ${ }^{11}{ }^{20}$. CHIT1 genotype was not available for this cohort, but the effect of this polymorphism on CSF CHIT1 levels might favour measurement of CHI3L2 instead, which showed similar diagnostic performance and clinical correlations but is without a similar confounding polymorphism.

Intriguingly, the analysis of clinical and biochemical correlations described here demonstrates differences in the relationship between the three chitinase proteins and different clinical and biochemical parameters. Specifically, CHIT1 and CHI3L2 correlated with the rate of disease progression, whereas CHI3L1 correlated with clinical upper motor neuron burden and cognitive impairment. This is in keeping previous studies demonstrating elevated CSF CHI3L1 in patients with sporadic FTD compared with ALS (although this was not recapitulated in familial FTD) and elevated CHIT1 in ALS compared with familial FTD ${ }^{11}$.

These findings hint at differences in the source of CHIT1, CHI3L2 and CHI3L1 within the central nervous system in ALS. Although CHIT1 colocalises with microglia in the spinal cord of ALS patients ${ }^{9}$, similar findings have not been demonstrated in brain regions affected in ALS. In addition, although mRNA expression data shows that CHI3L1 and CHI3L2 are 
upregulated in the motor cortex of patients with ALS the cellular origin of this elevation has not been demonstrated. Studies in other diseases give a conflicting picture. In Alzheimer disease CHI3L1 colocalises with astrocytes but not microglia ${ }^{21}$, whereas in multiple sclerosis CHI3L1 is produced in microglia and astrocytes ${ }^{22}$. The secretion of CHI3L2 within the central nervous system has not been studied, though this work would suggest it is likely to be similar to CHIT1. Further work is required to establish the regional and cellular expression of these proteins in order to delineate their relevance to ALS pathogenesis.

This study did not demonstrate significant longitudinal change in the CSF levels of chitinase proteins. This is consistent with measurement of CSF and serum neurofilaments and the rateof-change of the ALSFRS-R, which also remain relatively stable over time and suggests that this reflects a relatively stable immune response through the course of the disease ${ }^{23}$. Whether this response is a primary driver of progression, a contributory or protective factor requires additional work.

The levels of all three chitinase proteins were low in AGCs, in keeping with studies examining CSF CHIT1 levels in this group (though it must be noted that in the current study the number of subjects is small and younger than the control group $)^{11}$. Together, these data suggest that CSF glial activity is likely to normal in the years before the onset of symptoms in ALS. Greater insight into the glial response just prior to the symptomatic phase would be gained from examination of longitudinal samples from subjects transitioning from asymptomatic gene carriage to symptomatic ALS, as has been undertaken using neurofilament proteins ${ }^{24}$.

Similarly, although a detrimental role for excessive glial activity is suggested by some animal models of ALS, it remains unresolved whether the correlation between disease severity and microglial activity in humans is a factor modulating the underlying progression of ALS or is simply a bystander effect ${ }^{717}$. The finding of a correlation between survival and CHIT1 level independent of pNFH lends limited support to a modifying role of microglia, though additional validation of this is necessary particularly given the omission of certain prognostic factors for which data was unavailable.

Although this study suggests that CSF chitinase proteins do not improve upon neurofilaments for the diagnosis of ALS, it suggests that they might find adjunctive use in the prediction of progression and survival and in particular that they represent a useful biochemical measure of the effect of therapeutic strategies targeting glial responses.

\section{CONTRIBUTORSHIP STATEMENT}

All authors contributed to the conception and design of the study and data acquisition. AGT and EG contributed to drafting the text and preparing the figures.

\section{COMPETING INTERESTS AND FUNDING}

The authors declare no competing interests. MRT receives funding from the Medical Research Council \& Motor Neurone Disease Association Lady Edith Wolfson Senior Clinical Fellowship MR/K01014X/1. 


\section{REFERENCES}

1. Brown RH, Jr., Al-Chalabi A. Amyotrophic Lateral Sclerosis. N Engl J Med 2017;377(16):1602. doi: 10.1056/NEJMc1710379

2. Talbot K, Feneberg E, Scaber J, et al. Amyotrophic lateral sclerosis: the complex path to precision medicine. J Neurol 2018 doi: 10.1007/s00415-018-8983-8

3. Neumann M, Sampathu DM, Kwong LK, et al. Ubiquitinated TDP-43 in frontotemporal lobar degeneration and amyotrophic lateral sclerosis. Science 2006;314(5796):130-3. doi: 10.1126/science.1134108

4. Byrne S, Walsh C, Lynch C, et al. Rate of familial amyotrophic lateral sclerosis: a systematic review and meta-analysis. J Neurol Neurosurg Psychiatry 2011;82(6):6237. doi: $10.1136 /$ jnnp.2010.224501

5. Ringholz GM, Appel SH, Bradshaw M, et al. Prevalence and patterns of cognitive impairment in sporadic ALS. Neurology 2005;65(4):586-90. doi: 10.1212/01.wnl.0000172911.39167.b6

6. Zou ZY, Zhou ZR, Che CH, et al. Genetic epidemiology of amyotrophic lateral sclerosis: a systematic review and meta-analysis. J Neurol Neurosurg Psychiatry 2017;88(7):54049. doi: 10.1136/jnnp-2016-315018

7. Brettschneider J, Toledo JB, Van Deerlin VM, et al. Microglial activation correlates with disease progression and upper motor neuron clinical symptoms in amyotrophic lateral sclerosis. PLoS One 2012;7(6):e39216. doi: 10.1371/journal.pone.0039216

8. Thompson AG, Gray E, Thezenas ML, et al. Cerebrospinal fluid macrophage biomarkers in amyotrophic lateral sclerosis. Ann Neurol 2018;83(2):258-68. doi: 10.1002/ana.25143

9. Steinacker P, Verde F, Fang L, et al. Chitotriosidase (CHIT1) is increased in microglia and macrophages in spinal cord of amyotrophic lateral sclerosis and cerebrospinal fluid levels correlate with disease severity and progression. J Neurol Neurosurg Psychiatry 2018;89(3):239-47. doi: 10.1136/jnnp-2017-317138

10. Varghese AM, Sharma A, Mishra P, et al. Chitotriosidase - a putative biomarker for sporadic amyotrophic lateral sclerosis. Clin Proteomics 2013;10(1):19. doi: 10.1186/1559-0275-10-19

11. Oeckl P, Weydt P, Steinacker $P$, et al. Different neuroinflammatory profile in amyotrophic lateral sclerosis and frontotemporal dementia is linked to the clinical phase. J Neurol Neurosurg Psychiatry 2018 doi: 10.1136/jnnn-2018-318868

12. Kester MI, Teunissen CE, Sutphen C, et al. Cerebrospinal fluid VILIP-1 and YKL-40, candidate biomarkers to diagnose, predict and monitor Alzheimer's disease in a memory clinic cohort. Alzheimers Res Ther 2015;7(1):59. doi: 10.1186/s13195-0150142-1

13. Ryberg H, An J, Darko S, et al. Discovery and verification of amyotrophic lateral sclerosis biomarkers by proteomics. Muscle Nerve 2010;42(1):104-11. doi: 10.1002/mus.21683

14. BenGershom E, Briggeman-Mol GJ, de Zegher F. Cerebrospinal fluid C-reactive protein in meningitis: diagnostic value and pathophysiology. Eur J Pediatr 1986;145(4):246-9.

15. Moore DH, 2nd, Miller RG. Improving efficiency of ALS clinical trials using lead-in designs. Amyotroph Lateral Scler Other Motor Neuron Disord 2004;5 Suppl 1:57-60. doi: 10.1080/17434470410019997 
16. Abrahams S, Newton J, Niven E, et al. Screening for cognition and behaviour changes in ALS. Amyotroph Lateral Scler Frontotemporal Degener 2014;15(1-2):9-14. doi: 10.3109/21678421.2013.805784

17. Turner MR, Cagnin A, Turkheimer FE, et al. Evidence of widespread cerebral microglial activation in amyotrophic lateral sclerosis: an [11C](R)-PK11195 positron emission tomography study. Neurobiol Dis 2004;15(3):601-9. doi: 10.1016/j.nbd.2003.12.012

18. Westeneng HJ, Debray TPA, Visser AE, et al. Prognosis for patients with amyotrophic lateral sclerosis: development and validation of a personalised prediction model. Lancet Neurol 2018;17(5):423-33. doi: 10.1016/S1474-4422(18)30089-9

19. Pagliardini V, Pagliardini S, Corrado L, et al. Chitotriosidase and lysosomal enzymes as potential biomarkers of disease progression in amyotrophic lateral sclerosis: a survey clinic-based study. J Neurol Sci 2015;348(1-2):245-50. doi: 10.1016/j.jns.2014.12.016

20. Boot RG, Renkema GH, Verhoek M, et al. The human chitotriosidase gene. Nature of inherited enzyme deficiency. J Biol Chem 1998;273(40):25680-5.

21. Querol-Vilaseca M, Colom-Cadena M, Pegueroles J, et al. YKL-40 (Chitinase 3-like I) is expressed in a subset of astrocytes in Alzheimer's disease and other tauopathies. $J$ Neuroinflammation 2017;14(1):118. doi: 10.1186/s12974-017-0893-7

22. Hinsinger G, Galeotti N, Nabholz N, et al. Chitinase 3-like proteins as diagnostic and prognostic biomarkers of multiple sclerosis. Mult Scler 2015;21(10):1251-61. doi: 10.1177/1352458514561906

23. Lu CH, Macdonald-Wallis C, Gray E, et al. Neurofilament light chain: A prognostic biomarker in amyotrophic lateral sclerosis. Neurology 2015;84(22):2247-57. doi: 10.1212/WNL.0000000000001642

24. Benatar M, Wuu J, Andersen PM, et al. Neurofilament light: A candidate biomarker of presymptomatic amyotrophic lateral sclerosis and phenoconversion. Ann Neurol 2018;84(1):130-39. doi: 10.1002/ana.25276 
Table 1 Demographic table. $p$-values for $\chi^{2}$ test (categorical data) or Kruskal-Wallis $H$ test (continuous data; or Mann-Whitney U test for two-group comparisons). ${ }^{*}$ Post hoc pairwise Fisher exact tests with Benjamini-Hochberg FDR cor- rection indicated significantly more male subjects in ALS and mimics compared to other groups. ${ }^{+}$Dunn's post hoc with Benjamini-Hochberg FDR correction $p<0.05$ for asymptomatic gene carriers versus other groups. ALS - amyotrophic lateral sclerosis; PLS - primary lateral sclerosis.

\begin{tabular}{|l|c|c|c|c|c|c|}
\hline & ALS & PLS & $\begin{array}{c}\text { Mimic } \\
\text { conditions }\end{array}$ & $\begin{array}{c}\text { Healthy } \\
\text { control }\end{array}$ & $\begin{array}{c}\text { Asympto- } \\
\text { matic gene } \\
\text { carriers }\end{array}$ & $p$ \\
\hline$n$, first visit & 82 & 10 & 12 & 25 & 5 & - \\
\hline$n$, second visit & 33 & 5 & - & - & - & - \\
\hline$n$, third visit & 19 & 3 & - & - & - & - \\
\hline$n$, fourth visit & 10 & 2 & - & - & - & - \\
\hline$n$, fifth visit & 2 & - & - & - & - & - \\
\hline Male, $n$ (\%) & $63(76.8)$ & $4(40)$ & $11(91.7)$ & $12(48)$ & $1(20)$ & $0.013^{*}$ \\
\hline $\begin{array}{l}\text { Age at sampling, } \\
\text { mean } \pm \text { SD }\end{array}$ & $\begin{array}{c}59.9 \pm \\
10.7\end{array}$ & $66.4 \pm 9.7$ & $\begin{array}{c}57.7 \pm \\
15.5\end{array}$ & $56.5 \pm 9.2$ & $39.7 \pm 7.1$ & $0.011^{+}$ \\
\hline $\begin{array}{l}\text { Age at onset, mean } \\
\pm \text { SD }\end{array}$ & $\begin{array}{c}56.8 \pm \\
11.2\end{array}$ & $\begin{array}{c}44.0 \pm \\
26.0\end{array}$ & - & - & - & 0.010 \\
\hline Bulbar onset, $n(\%)$ & $22(26.8)$ & $1(10)$ & - & - & - & 0.438 \\
\hline $\begin{array}{l}\text { Disease progression } \\
\text { rate median [IQR] }\end{array}$ & $\begin{array}{c}0.45[0.23- \\
0.60]\end{array}$ & $\begin{array}{c}0.12[0.08- \\
0.17]\end{array}$ & - & - & - & 0.040 \\
\hline Deaths, $n$ (\%) & $44(54)$ & 0 & & & & $<0.001$ \\
\hline
\end{tabular}


Table 2 Cross-sectional chitinase levels. Median [IQR], ng/mL. CHIT1 - Chitotriosidase-1; CHI3L1 - Chitinase 3-like protein 1; CHI3L2 - Chitinase 3-like protein 2; pNFH phosphorylated Neurofilament heavy chain; ALS - amyotrophic lateral sclerosis; PLS primary lateral sclerosis.

\begin{tabular}{|l|c|c|c|c|}
\hline & CHIT1 & CHI3L1 & CHI3L2 & pNFH \\
\hline ALS & $\begin{array}{c}8.53[4.43- \\
17.16]\end{array}$ & $\begin{array}{c}183.82[141.94- \\
243.35]\end{array}$ & $\begin{array}{c}16.12[10.97- \\
21.84]\end{array}$ & $2.60[1.77-4.06]$ \\
\hline Mimic & $1.33[1.19-4.66]$ & $\begin{array}{c}132.32[89.24- \\
168.87]\end{array}$ & $7.34[6.43-8.11]$ & $0.51[0.38-0.72]$ \\
\hline PLS & $\begin{array}{c}1.76[0.91- \\
10.43]\end{array}$ & $\begin{array}{c}175.79[113.77- \\
213.07]\end{array}$ & $8.84[7.52-9.52]$ & $0.62[0.51-1.00]$ \\
\hline $\begin{array}{l}\text { Healthy } \\
\text { control }\end{array}$ & $0.97[0.57-1.93]$ & $\begin{array}{c}123.76[81.36- \\
151.41]\end{array}$ & $6.48[4.73-8.07]$ & $0.35[0.31-0.47]$ \\
\hline $\begin{array}{l}\text { Asymptomatic } \\
\text { gene carrier }\end{array}$ & $0.96[0.93-1.56]$ & $\begin{array}{c}69.29[69.21- \\
88.30]\end{array}$ & $5.92[4.99-6.18]$ & $0.22[0.21-0.22]$ \\
\hline
\end{tabular}


Table 3 Multiple regression modelling of log-transformed disease progression rate. CHIT1 Chitotriosidase-1; CHI3L1 - Chitinase 3-like protein 1; CHI3L2 - Chitinase 3-like protein 2; pNFH - phosphorylated Neurofilament heavy chain.

\begin{tabular}{|c|c|c|c|c|c|c|c|c|c|c|c|}
\hline & \multicolumn{3}{|c|}{ CHIT1 } & \multicolumn{3}{|c|}{ CHI3L1 } & \multicolumn{3}{|c|}{ CHI3L2 } & \multicolumn{2}{|r|}{ pNFH } \\
\hline & $\begin{array}{c}\text { Gradie } \\
\text { nt }\end{array}$ & CI & $p$ & $\begin{array}{c}\text { Gradie } \\
\text { nt }\end{array}$ & CI & $p$ & $\begin{array}{c}\text { Gradie } \\
\text { nt }\end{array}$ & CI & $p$ & $\begin{array}{c}\text { Gradie } \\
\text { nt }\end{array}$ & CI \\
\hline $\begin{array}{l}\log _{10} \text { chitinase } \\
\text { protein/pNFH }\end{array}$ & 0.264 & $\begin{array}{c}0.002- \\
0.527\end{array}$ & 0.049 & 0.621 & $\begin{array}{l}-0.234- \\
1.526\end{array}$ & 0.176 & 0.620 & $\begin{array}{c}0.005- \\
1.234\end{array}$ & 0.048 & 1.069 & $\begin{array}{c}0.649- \\
1.489\end{array}$ \\
\hline Sex (male) & 0.041 & $\begin{array}{c}-0.234- \\
0.326 \\
\end{array}$ & 0.774 & -0.068 & $\begin{array}{l}-0.400- \\
0.264 \\
\end{array}$ & 0.684 & -0.114 & $\begin{array}{c}-0.440- \\
0.211 \\
\end{array}$ & 0.486 & -0.188 & $\begin{array}{r}-0.497 \\
-0.121 \\
\end{array}$ \\
\hline $\begin{array}{l}\text { Site of onset } \\
\text { (limb) }\end{array}$ & 0.133 & $\begin{array}{c}-0.178- \\
0.444\end{array}$ & 0.398 & 0.200 & $\begin{array}{l}-0.170- \\
0.571\end{array}$ & 0.285 & 0.147 & $\begin{array}{c}-0.204- \\
0.500\end{array}$ & 0.406 & 0.276 & $\begin{array}{c}-0.075 \\
- \\
0.626\end{array}$ \\
\hline $\begin{array}{l}\text { Age of onset } \\
\text { (years) }\end{array}$ & 0.014 & $\begin{array}{c}0.005- \\
0.023\end{array}$ & 0.004 & 0.015 & $\begin{array}{c}0.004- \\
0.026\end{array}$ & 0.008 & 0.014 & $\begin{array}{c}0.0003- \\
0.025\end{array}$ & 0.011 & 0.001 & $\begin{array}{c}-0.02- \\
0.019\end{array}$ \\
\hline & \multicolumn{2}{|c|}{0.10} & 0.015 & \multicolumn{2}{|c|}{0.07} & 0.041 & \multicolumn{2}{|c|}{0.10} & 0.014 & \multicolumn{2}{|c|}{0.29} \\
\hline
\end{tabular}


Table 4 Cox proportional hazards survival modelling of factors known to be associated with survival and chitinase proteins without and with inclusion of CSF pNFH. CSF cerebrospinal fluid; HR - hazard ratio; CI - confidence interval; CHIT1 - Chitotriosidase-1; CHI3L1 - Chitinase 3-like protein 1; CHI3L2 - Chitinase 3-like protein 2; pNFH phosphorylated Neurofilament heavy chain.

\begin{tabular}{|l|c|c|c|c|c|c|}
\hline & HR & $95 \%$ CI & $p$ & HR & $95 \%$ CI & $p$ \\
\hline Gender (male) & 0.643 & $0.230-1.794$ & 0.398 & 0.526 & $0.175-1.574$ & 0.251 \\
\hline Onset site (spinal) & 1.824 & $0.421-7.895$ & 0.421 & 2.753 & $0.430-17.607$ & 0.285 \\
\hline Age at onset (years) & 1.105 & $1.023-1.192$ & 0.011 & 1.090 & $1.011-1.176$ & 0.025 \\
\hline CHIT1 & 11.684 & $2.620-52.101$ & 0.001 & 6.831 & $1.135-34.687$ & 0.021 \\
\hline CHI3L1 & 1.130 & $0.031-40.980$ & 0.947 & 1.929 & $0.035-105.765$ & 0.748 \\
\hline CHI3L2 & 0.058 & $0.001-2.228$ & 0.126 & 0.012 & $0.000-1.310$ & 0.065 \\
\hline pNFH & - & - & - & 1.338 & $0.917-1.011$ & 0.118 \\
\hline
\end{tabular}




\section{Figure 1}

A-C Cross-sectional levels of CSF CHIT1, CHI3L1 and CHI3L2. D-F receiver operator characteristic analysis of chitinase proteins, pNFH and the optimum combination of chitinase proteins and pNFH. CSF - cerebrospinal fluid; ALS - amyotrophic lateral sclerosis; Mim mimic conditions; PLS - primary lateral sclerosis; HC - healthy control; AGC asymptomatic gene carrier; CHIT1 - Chitotriosidase-1; CHI3L1 - Chitinase 3-like protein 1; CHI3L2 - Chitinase 3-like protein 2; pNFH - phosphorylated Neurofilament heavy chain; AUC - area under the curve.
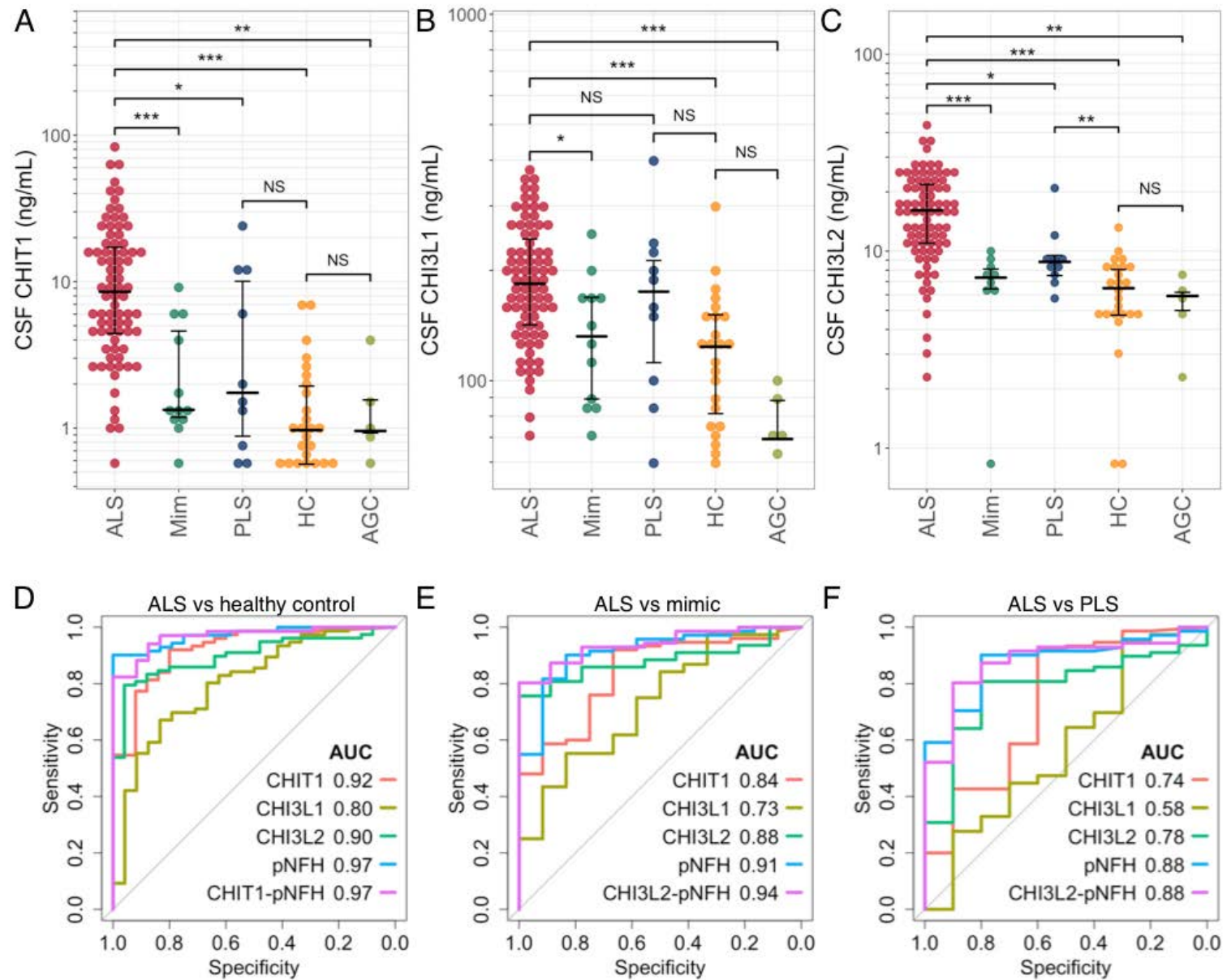
Figure 2 Correlation of CSF chitinase protein and pNFH levels with the rate of disease progression (A-D), cognitive function as measured by the ALS-specific ECAS score (E-H) and upper motor neuron score (I-L). Pearson's $r$ given. CSF - cerebrospinal fluid; ALS amyotrophic lateral sclerosis; PLS - primary lateral sclerosis; PR - disease progression rate; ECAS - Edinburgh cognitive and behavioural ALS screen; CHIT1 - Chitotriosidase-1; CHI3L1 - Chitinase 3-like protein 1; CHI3L2 - Chitinase 3-like protein 2; pNFH phosphorylated Neurofilament heavy chain.
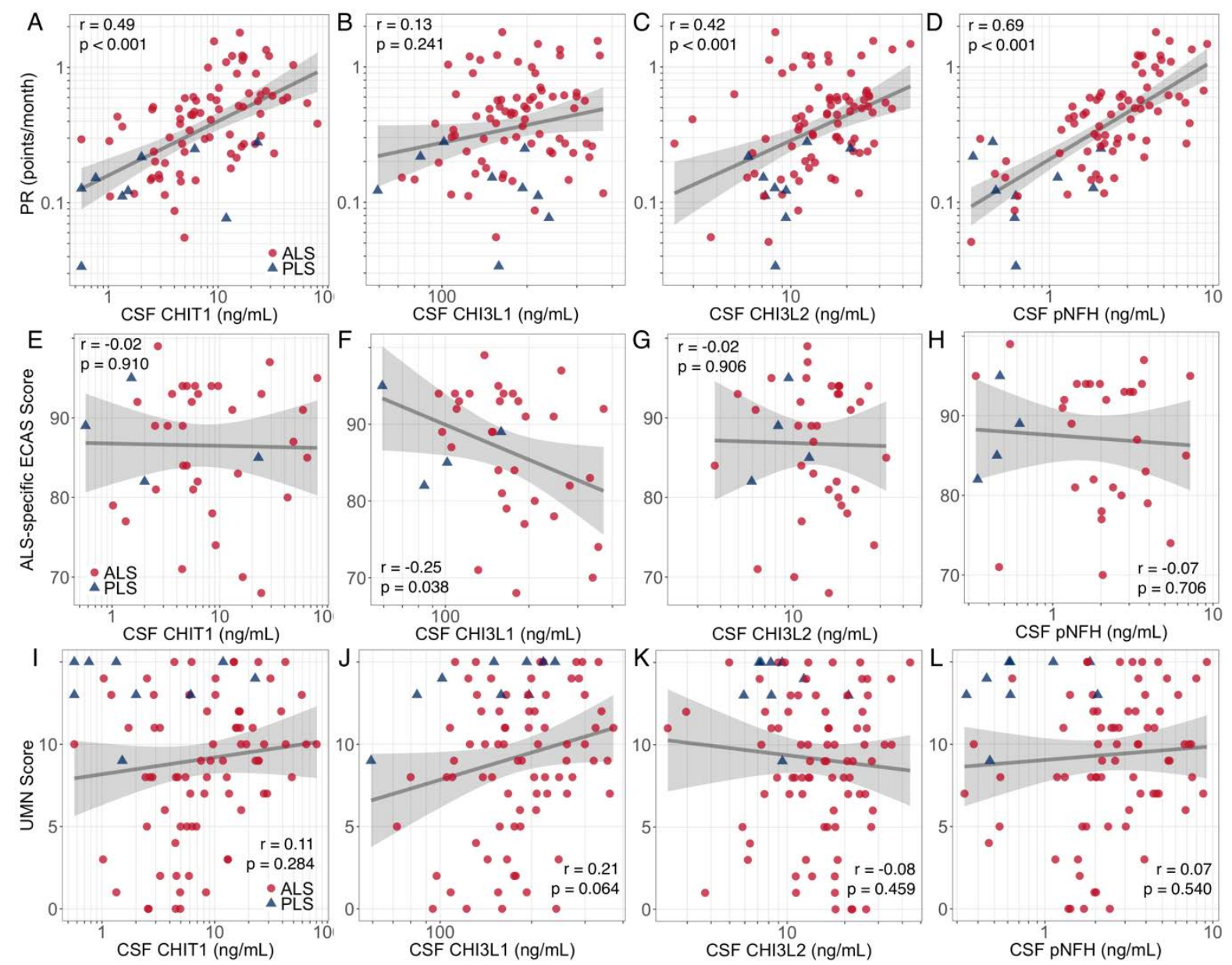
Figure 3 Longitudinal stability of the CSF chitinase proteins. Thick, shaded line indicates the overall linear fit of high and low disease progression rate strata. CSF - cerebrospinal fluid; ALS - amyotrophic lateral sclerosis; PR - disease progression rate; CHIT1 Chitotriosidase-1; CHI3L1 - Chitinase 3-like protein 1; CHI3L2 - Chitinase 3-like protein 2.
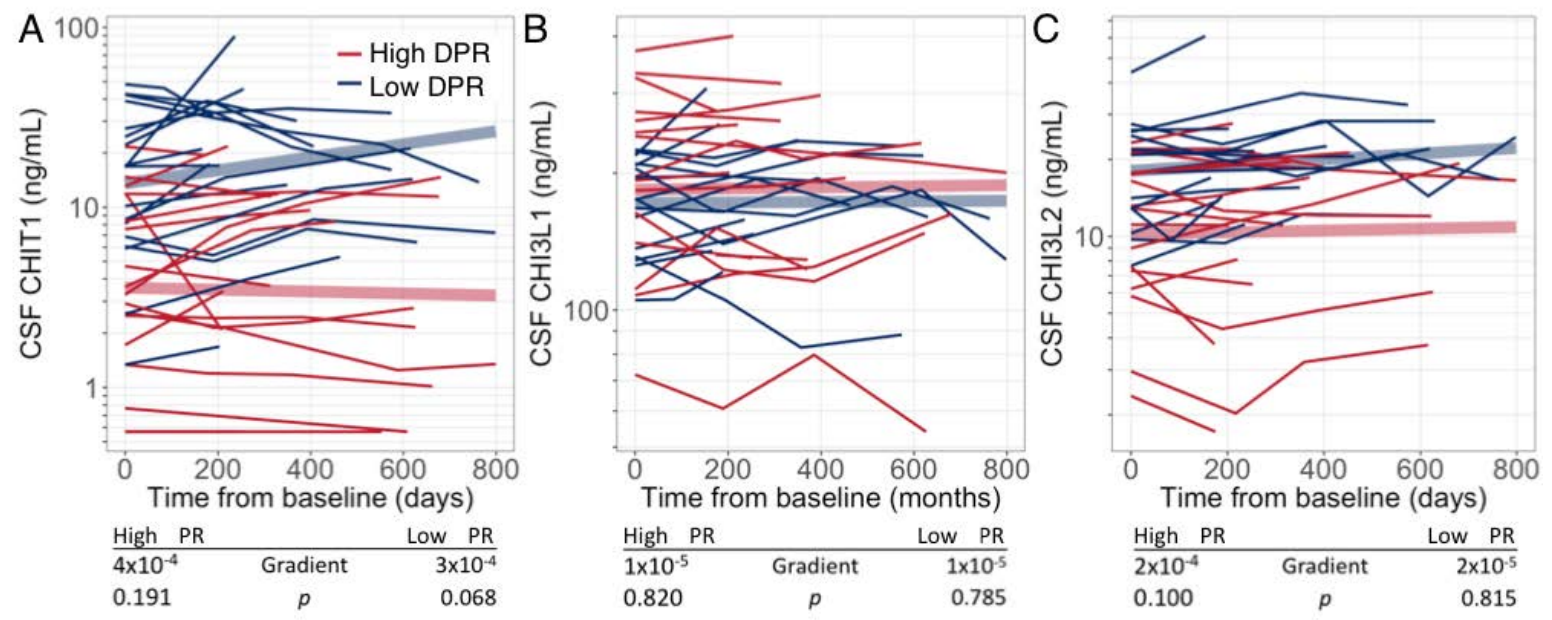
Figure 4 Serum chitinase protein levels. A-C relationship between CSF and serum chitinase protein levels. Thick grey line shows linear regression fit, ribbon indicates $95 \%$ confidence interval. Black line indicates CSF=serum (points above line indicate higher levels in serum than CSF). D-F comparison of serum chitinase protein levels between ALS and healthy control subjects. No significant difference is observed for any chitinase protein. CSF cerebrospinal fluid; ALS - amyotrophic lateral sclerosis; HC - healthy control; CHIT1 Chitotriosidase 1; CHI3L1 - Chitinase 3-like protein 1; CHI3L2 - Chitinase 3-like protein 2.
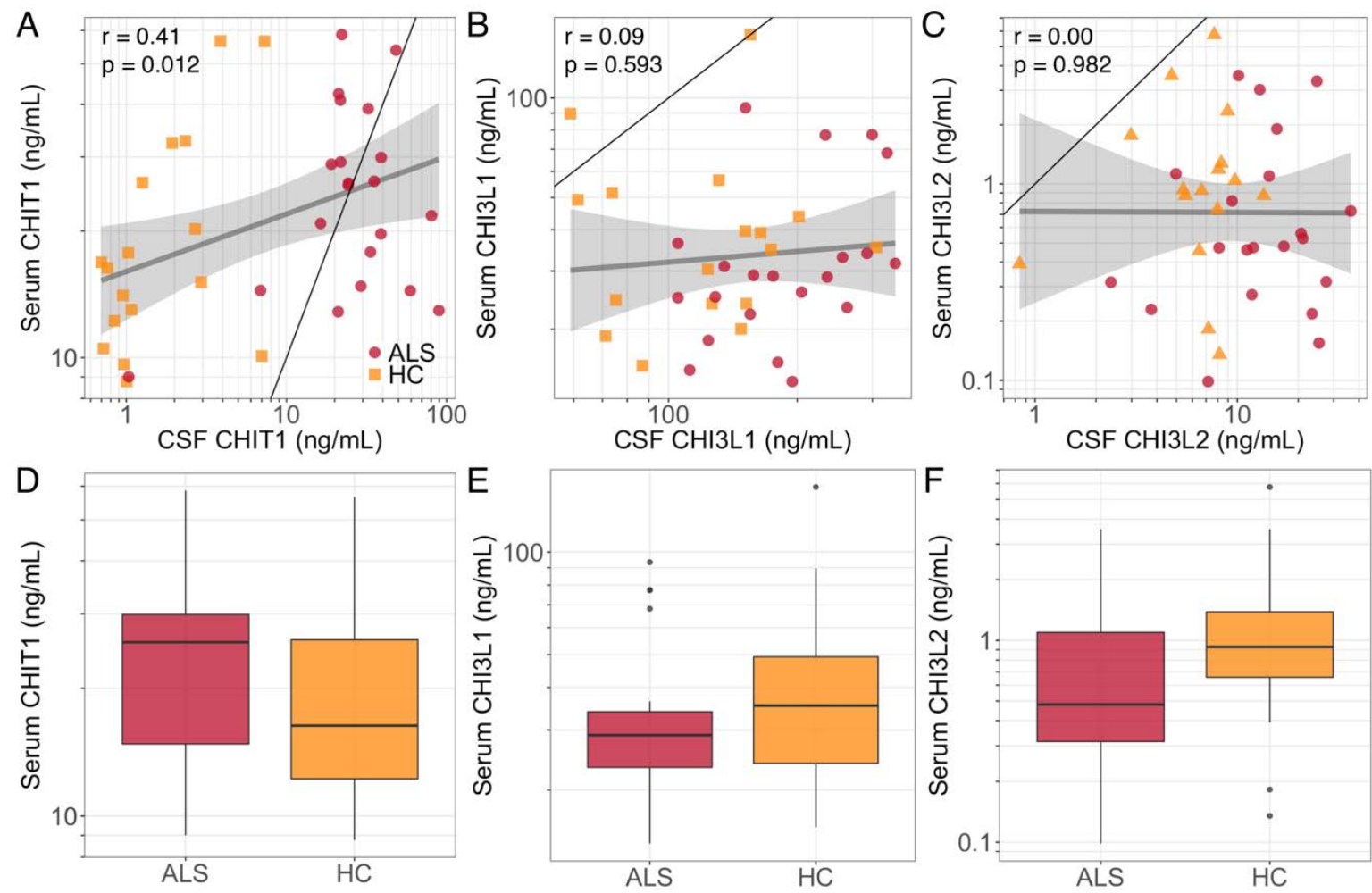